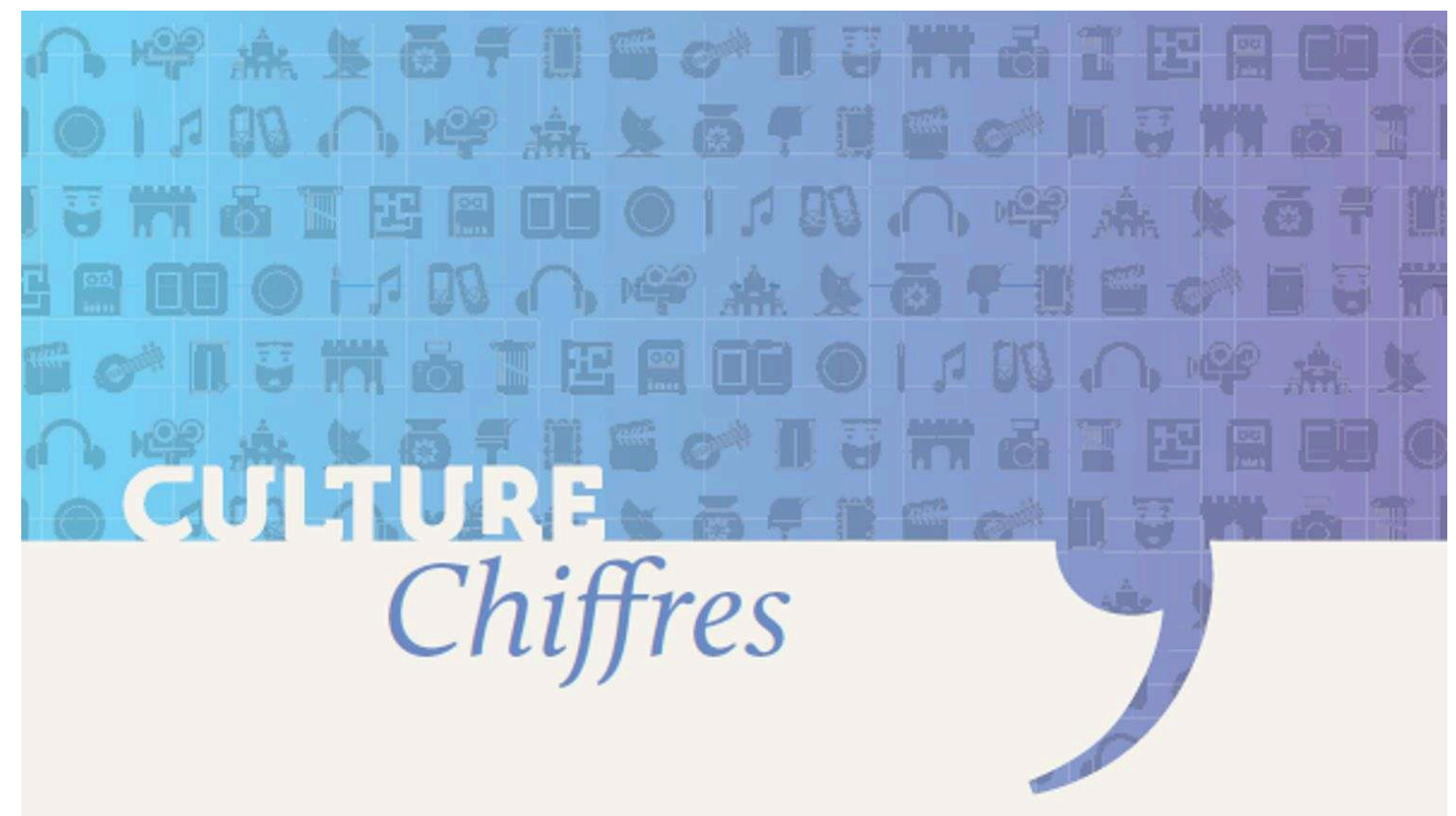

Vingt ans d'évolution de l'emploi dans les professions culturelles

I99I-2OII

Marie Gouyon

Frédérique Patureau

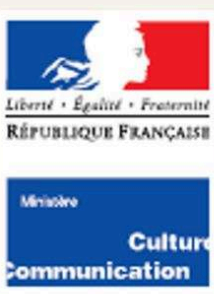

2014-6

Ministère de la Culture et de la Communication

Département des études,

de la prospective

et des statistiques 


\section{Vingt ans d'évolution de l'emploi dans les professions culturelles (1991-2011)}

Twenty Years of Changing Employment Patterns in the Cultural Professions (1991-2011)

Frédérique Patureau et Marie Gouyon

Éditeur : Département des études, de la prospective et des statistiques

Lieu d'édition : Paris

Année d'édition : 2014

Date de mise en ligne : 21 septembre 2015

Collection : Culture chiffres

ISBN électronique : 9782111398214

\section{C books}

http://books.openedition.org

\section{Édition imprimée}

Date de publication : 1 octobre 2014

Nombre de pages : 24

\section{Référence électronique}

PATUREAU, Frédérique ; GOUYON, Marie. Vingt ans d'évolution de l'emploi dans les professions culturelles (1991-2017). Nouvelle édition [en ligne]. Paris : Département des études, de la prospective et des statistiques, 2014 (généré le 25 avril 2021). Disponible sur Internet : <http://books.openedition.org/ deps/457>. ISBN : 9782111398214 


\title{
Vingt ans d'évolution de l'emploi dans les professions culturelles (I99I-2OII)
}

\author{
Marie Gouyon, Frédérique Patureau*
}

Au cours des vingt dernières années, les effectifs en emploi des professions culturelles ont connu une expansion exceptionnelle, bien plus importante que celle de l'ensemble des actifs.

Le profil des personnes qui exercent ces professions et celui des emplois qu'elles occupent présentaient, au début des années 1990 tout comme aujourd'hui, certaines caractéristiques d'ensemble qui distinguent cette population professionnelle du reste des actifs, au-delà de la grande diversité des métiers qu'elle rassemble. C'est ainsi une population plutôt plus jeune que la moyenne des actifs, plus masculine, nettement plus diplômée et plus francilienne. L'emploi y est globalement marqué à la fois par le poids important, constant depuis vingt ans, du non-salariat (trois fois plus que dans l'ensemble de la population active) et par une plus grande flexibilité du salariat (davantage de contrats courts, de temps partiel et de pluriactivité).

Pour autant, les professions culturelles ne sont pas restées imperméables à certaines évolutions qui ont marqué l'ensemble du monde du travail depuis le début des années 1990. Le mouvement continu de féminisation des emplois, par exemple, s'y est opéré dans les mêmes proportions que dans l'ensemble de la population active, amenant certaines professions culturelles traditionnellement très masculines à connaître un doublement de la part de leurs effectifs féminins en vingt ans.

En outre, les efforts des politiques publiques de décentralisation ont conduit à une légère atténuation de la concentration francilienne de ces professions. D'autres évolutions communes à l'ensemble des actifs comme l'élévation continue du niveau de diplôme et le développement de la flexibilité de l'emploi - deux caractéristiques attachées de longue date au monde de l'emploi culturel - ont continué à s'y développer, dans des proportions toujours supérieures à celles observées dans le reste de la population active.

* Département des études, de la prospective et des statistiques. 
Professions des spectacles et des arts visuels, métiers d'art, journalistes, écrivains, conservateurs, documentalistes, architectes, professeurs d'art, etc. : les professions culturelles $^{1}$ (voir encadré, $\S 3$, p. 20) constituent un ensemble particulièrement hétérogène du point de vue de leurs effectifs, mais aussi de l'encadrement juridique dont elles font l'objet, des statuts d'emploi dans lesquels elles s'exercent, ou encore des compétences et savoir-faire qu'elles mobilisent.

Elles ont cependant pour point commun de s'être considérablement développées au cours des vingt dernières années (graphique 1).

Au début des années 1990, 381000 personnes déclaraient exercer à titre principal une profession culturelle. Vingt ans plus tard, elles sont 573000 , soit $50 \%$ de plus (tableau 1). Dans le même temps, l'ensemble de la population active en emploi a augmenté de façon nettement plus modérée (+ $16 \%$ ). La part des professions culturelles dans l'ensemble des actifs en emploi s'est donc accrue sensiblement et atteint 2,2\% en 2011.

\section{Graphique 1 - Évolution des effectifs en emploi dans les professions culturelles et dans l'ensemble des actifs, 1991-2011}

Indice 100 en 1991

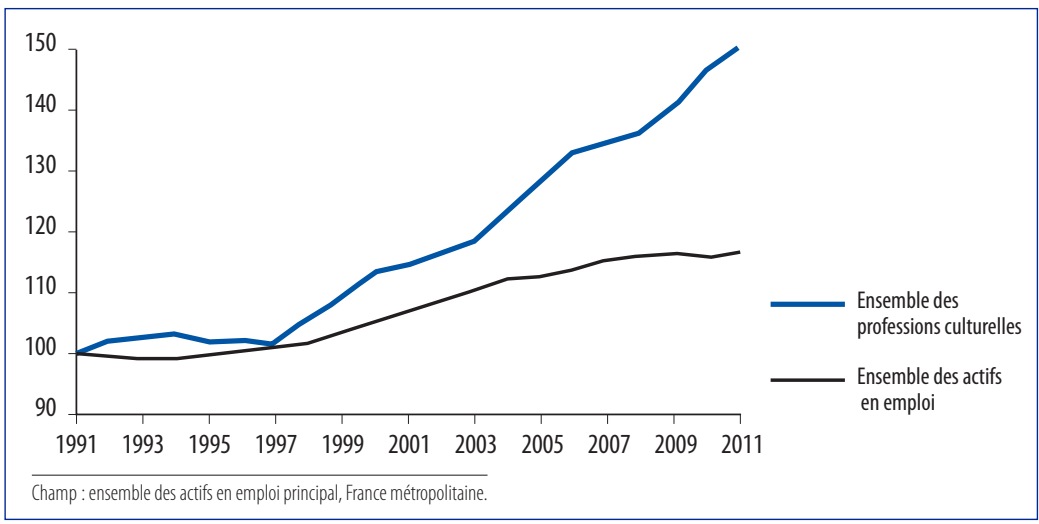

Source: Enquêtes Emploi, Insee/DEPs, Ministère de la Culture et de la Communication, 2014

\section{Forte expansion des professions culturelles}

Toutes les professions culturelles - ou presque - sont concernées par cet essor et en tout premier lieu celles du spectacle, dont l'effectif a pratiquement doublé pour atteindre 166000 professionnels en 2011 (tableau 1). Ce développement, lui-même porté par la montée en puissance du régime de l'intermittence ${ }^{2}$, a concerné les professionnels techniques (techniciens vidéo, réalisateurs, chefs opérateurs, ingénieurs du son, monteurs...) plus encore que les artistes (musiciens, danseurs, comédiens, artistes de cirque...).

1. Par souci de simplification, le terme de «profession » est employé ici de façon générique (et indifféremment à celui de « métier »), essentiellement par référence à la nomenclature des professions et catégories socioprofessionnelles (PCS) qui sert de cadre à la présente analyse (voir aussi encadré, § 3, p. 20).

2. Marie Gouyon, Frédérique Patureau, Tendances de l'emploi dans le spectacle, Paris, Ministère de la Culture et de la Communication, DEPS, coll. «Culture chiffres », 2014-2, 2014. 
Tableau 1 - Effectifs en emploi en 1991 et 2011

\begin{tabular}{|lcc|c|}
\hline Professions & $\mathbf{1 9 9 1}$ & $\mathbf{2 0 1 1}$ & Variation (en \%) \\
\hline Professions des arts visuels et métiers d'art & 134500 & 194300 & +44 \\
Artistes plasticiens & 24700 & 29900 & +21 \\
Photographes & 16600 & 19900 & +20 \\
Professions des arts graphiques, de la mode et de la décoration & 51300 & 114300 & +123 \\
Métiers d'art & 41900 & 30200 & -28 \\
\hline Professions des spectacles & 85300 & 166000 & +95 \\
Artistes des spectacles & 37600 & 62600 & +66 \\
Professionnels technico-artistiques des spectacles & 47700 & 103400 & +117 \\
\hline Professions littéraires & 56100 & 88600 & +58 \\
Journalistes et cadres de l'édition & 40000 & 62800 & +57 \\
Auteurs littéraires et traducteurs & 16100 & 25800 & +60 \\
\hdashline Professions de l'archivage, de la conservation & & & \\
et de la documentation & 41800 & 25900 & -38 \\
\hline Architectes & 35700 & 51700 & +45 \\
\hdashline Professeurs d'art & 27600 & 46500 & +68 \\
\hline Ensemble des professions culturelles & & & +16 \\
\hline Ensemble des actifs en emploi & 381000 & 573000 & +50 \\
\hline Champ : ensemble des actifs en emploi principal, France métropolitaine. & 22103100 & 25741600 & \\
\hline
\end{tabular}

Source : Enquêtes Emploi, Insee/DEPS, Ministère de la Culture et de la Communication, 2014

Les métiers des arts graphiques, de la mode et de la décoration (graphistes, stylistes, designers) se sont eux aussi considérablement développés au cours des deux décennies: leurs effectifs (114 300 en 2011) ont plus que doublé. Il s'agit de métiers « jeunes » dont le développement est allé de pair avec celui des activités économiques de design et de création graphique, tant en nombre d'entreprises qu'en termes de diversification des activités (design conseil, multimédia interactif, webdesign, animation $3 \mathrm{D}$, design sonore, jeux vidéo, etc. ${ }^{3}$ ) (graphique 2 ).

Les effectifs des métiers littéraires (journalistes, auteurs, traducteurs) et ceux de professeurs d'art ont connu également une progression importante (respectivement $+58 \%$ et $+68 \%$ ), supérieure à la progression moyenne observée dans l'ensemble du champ culturel. Plus modérée, la progression du nombre d'architectes, d'artistes plasticiens et même de photographes est toutefois encore supérieure à l'évolution moyenne des actifs au niveau national.

Seules deux familles de professions culturelles s'inscrivent en marge de ce mouvement d'expansion. Celle des métiers d'art (facteurs d'instruments de musique, ébénistes d'art, marqueteurs, maîtres verriers, etc.) dont le recul des effectifs traduit sans doute un mouvement plus général de déclin de l'artisanat et du travail manuel hautement qualifié au profit de modes de production reposant sur des procédés moins techniques que technologiques, assistés par ordinateur. Et celle des professions de l'archivage, de la conservation et de la documentation (- $38 \%$ ), fortement concernées elles aussi par le développement des technologies numériques et qui se caractérisent par un vieillissement important de leurs effectifs.

3. Voir l'Économie du design en France, Paris, DGCIS, 2010 ; Jean-Pierre DuRAND, Joyce SeBAG, Métiers du graphisme, Paris, Ministère de la Culture et de la Communication, DEPS, coll. "Questions de culture », 2011. 
Graphique 2 - Évolution des effectifs en emploi dans certaines professions culturelles, 1991-2011

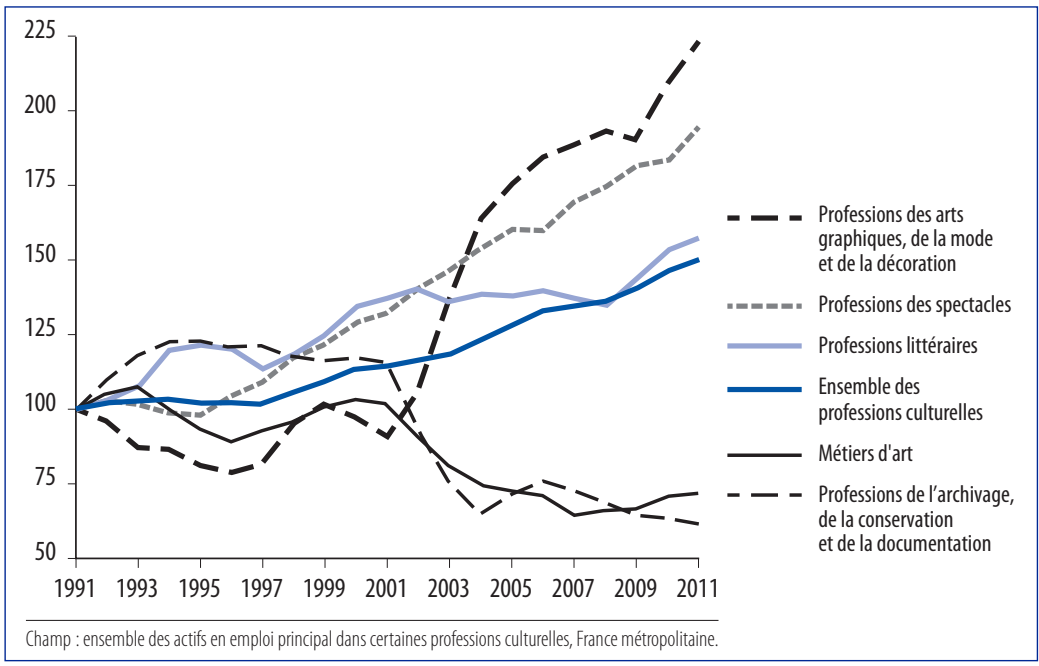

Source : Enquêtes Emploi, Insee/DEPS, Ministère de la Culture et de la Communication, 2014

\section{Un univers masculin qui s'ouvre lentement aux femmes}

En vingt ans, les professions culturelles se sont féminisées, comme l'ensemble des professions et dans des proportions comparables, mais restent en retrait de l'équilibre (quasi) paritaire atteint aujourd'hui dans l'ensemble de la population active ${ }^{4}$ : la part des femmes dans ce champ professionnel est ainsi passée de $39 \%$ au début des années 1990 à $43 \%$ en 2011, tandis qu'elle atteignait $48 \%$ dans l'ensemble de la population active au cours de cette même période (tableau 2 et graphique 3 ).

Dans le champ culturel, le mouvement de féminisation, même lorsqu'il a été très marqué, n'a pu combler le déficit féminin initial de certains métiers très peu féminisés en début de période. C'est le cas, en tout premier lieu, des métiers des spectacles, très masculins en début de période, et qui ne se sont que très faiblement féminisés: les métiers artistiques (36\% de femmes) et surtout techniques (31\%).

C'est le cas également d'autres métiers, comme les métiers d'art et le métier d'architecte. La part des femmes y a doublé en vingt ans, mais demeure encore modeste (respectivement $39 \%$ et $33 \%$ en 2011) et bien inférieure à celle qu'elles occupent dans l'ensemble du champ culturel.

Pour d'autres professions du champ culturel où la part des femmes était déjà significative en 1991, le mouvement de féminisation conduit aujourd'hui à une répartition des actifs proche de la parité (et parfois supérieure) : c'est le cas par exemple des professionnels des arts graphiques, de la mode et de la décoration (51\%), ou encore des journalistes et cadres de l'édition (45\%).

Les deux seuls groupes professionnels qui étaient très fortement féminisés au début des années 1990 ont connu des évolutions contrastées : le premier - les professions de

4. Marie Gouyon, Frédérique PATUREAU, la Lente Féminisation des professions culturelles (1991-2011), Paris, Ministère de la Culture et de la Communication, DEPS, coll. «Culture études » (à paraître en 2014). 
Tableau 2 - Caractéristiques sociodémographiques des actifs en emploi en 1991 et 2011

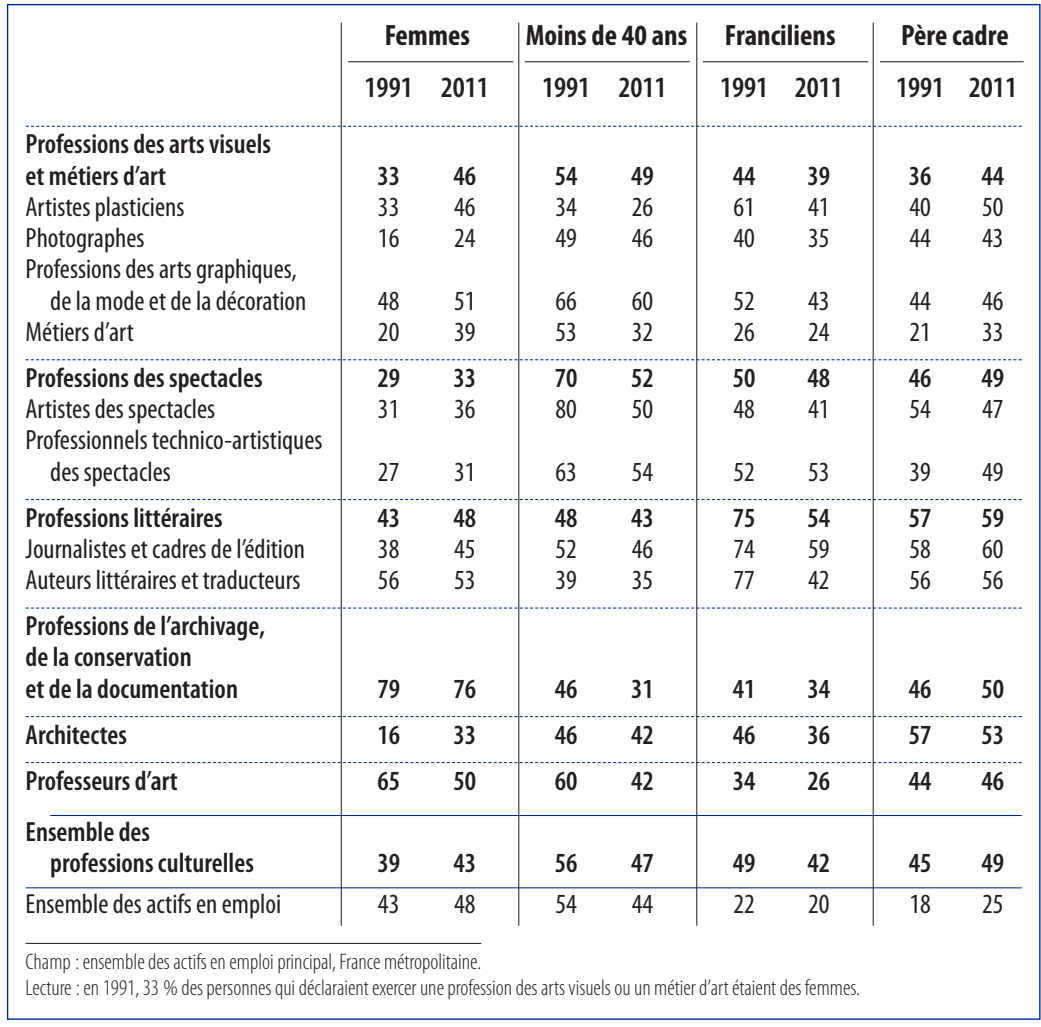

l'archivage, de la conservation et de la documentation - est resté massivement féminin, sans évolution notable vers la mixité (79\% de femmes en 1991, $76 \%$ en 2011); pour le second en revanche, les professeurs d'art, on observe une très nette masculinisation des emplois. Les effectifs de professeurs d'art (enseignants de musique, d'arts plastiques, etc., exerçant en dehors d'un établissement d'enseignement général) sont en effet désormais paritaires, ce qui constitue en soi une spécificité au sein des métiers de l'enseignement au niveau national où, par exemple, $81 \%$ des professeurs des écoles sont des femmes en 2011. Le rapprochement de cette évolution avec celle, concomitante, des conditions d'emploi dans ce métier - développement important des contrats courts et du travail à temps partiel - conforte l'hypothèse selon laquelle il s'agirait de plus en plus, pour les actifs concernés, d'un métier de complément à un métier de création ou d'interprétation artistique - métiers qui sont eux aussi très masculins. 


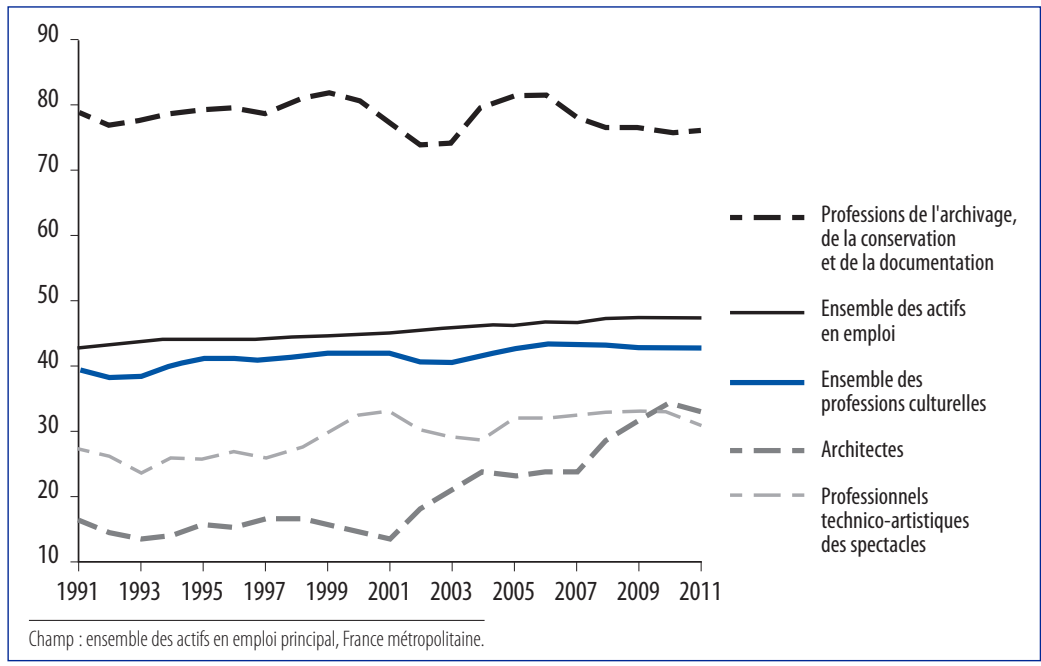

Source : Enquêtes Emploi, Insee/DEPS, Ministère de la Culture et de la Communication, 2014

\section{Davantage de jeunes mais aussi de seniors que la moyenne des actifs}

En lien avec la croissance des effectifs, avec la forte attractivité de ces métiers et avec le développement des formations spécialisées préparant à leur exercice, les actifs des professions culturelles sont plus jeunes que l'ensemble des actifs : $47 \%$ ont moins de 40 ans, contre $44 \%$ des actifs en moyenne (tableau 2 et graphique 4).

Les moins de 40 ans sont aujourd'hui particulièrement nombreux au sein des professions ayant connu récemment une forte croissance de leurs effectifs : professions des arts graphiques, de la mode et de la décoration (60\% de moins de 40 ans en 2011), professions des spectacles ( $54 \%$ de moins de 40 ans parmi les professions techniques).

À l'inverse, les jeunes sont minoritaires dans les métiers dont le développement a été plus modéré (et a fortiori, ceux dont les effectifs sont en recul) ou qui comptaient déjà une proportion importante de quadragénaires il y a vingt ans. Les moins de 40 ans représentent ainsi environ $45 \%$ des photographes, des journalistes, des architectes et des professeurs d'art. Ils ne représentent plus qu'un tiers, voire un quart, des artistes plasticiens, auteurs littéraires et professionnels de l'archivage, de la conservation et de la documentation.

Si elles demeurent globalement plus jeunes que la moyenne des actifs, les professions culturelles ont subi en vingt ans le même mouvement de vieillissement que celui observé dans l'ensemble de la population active (graphique 3). Cette tendance de fond est à rapprocher de l'allongement général de la durée des études et de la période de stabilisation dans la vie active, ainsi que de la progression du taux de chômage parmi les classes d'âge les plus jeunes. Toutes les professions culturelles sont concernées par ce phénomène, y compris celles qui se sont le plus développées. C'est le cas, notamment, des métiers du spectacle au sein desquels la part des jeunes 


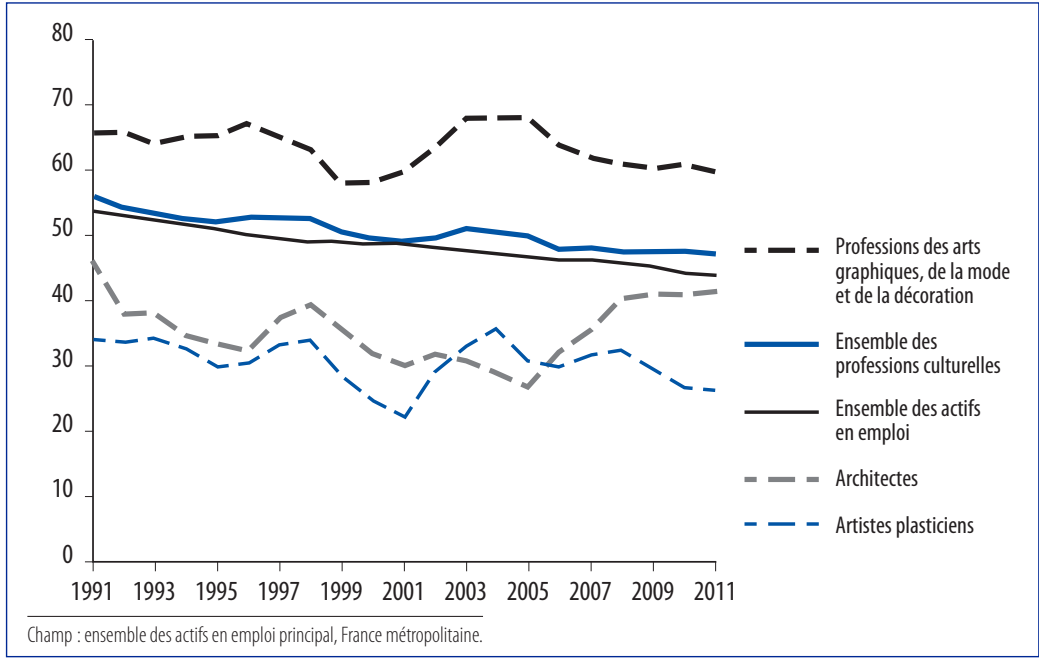

Source: Enquêtes Emploi, Insee/DEPS, Ministère de la Culture et de la Communication, 2014

demeure majoritaire, mais enregistre un recul de grande ampleur (- 18 points pour les métiers techniques et -30 points pour les artistes).

Cette tendance au vieillissement est à rapprocher également d'une autre caractéristique qui distingue cette fois les professions culturelles du reste des actifs : la part importante, et croissante, du nombre de professionnels encore en emploi à des âges avancés. Ainsi, en 2011,13\% des artistes plasticiens, $8 \%$ des auteurs littéraires et des traducteurs, $5 \%$ des architectes ont plus de 65 ans, tandis que cette classe d'âge ne représente qu'à peine $1 \%$ de l'ensemble des actifs en emploi. Cette spécificité tient sans doute pour partie à la surreprésentation des hauts niveaux de diplôme dans ces métiers (et, par voie de conséquence, à celle de personnes entrées tardivement sur le marché du travail après des études longues). Mais c'est aussi une caractéristique du travail indépendant ${ }^{5}$, particulièrement répandu dans ces métiers, et une spécificité des métiers de vocation, dans lesquels l'exercice professionnel repose sur un fort engagement personnel et identitaire.

Les professions culturelles se caractérisent donc à la fois par une proportion de jeunes plus importante que la moyenne et par un poids élevé de professionnels encore en emploi à des âges avancés.

5. Laurent TOULEMON, Situation professionnelle et comportements familiaux des indépendants, Paris, Insee, coll. «Économie et Statistique », n³19-320, décembre 1998. 


\section{Une concentration francilienne qui tend à s'atténuer}

En vingt ans, la concentration des actifs en région parisienne a accusé un recul plus important pour les professions culturelles que pour l'ensemble des actifs en emploi (tableau 2 et graphique 5). Le développement plus rapide des emplois culturels hors de l'île-de-France résulte des politiques actives de décentralisation conduites depuis les années 1980 et des actions de maillage du territoire en équipements culturels de toute nature (conservation, création, diffusion, enseignement, etc. ${ }^{6}$ ).

En dépit de cette régionalisation, $42 \%$ des personnes exerçant une profession culturelle habitent aujourd'hui en région parisienne, soit plus du double de l'ensemble de la population active en emploi. Cette concentration demeure particulièrement forte dans les professions qui travaillent majoritairement pour des structures de production elles-mêmes concentrées en Île-de-France : c'est le cas notamment des professionnels techniques des spectacles (53\%), très liés aux entreprises de production et de diffusion audiovisuelles (en 2010,63\% des entreprises de l'audiovisuel sont implantées en Île-de-France), et aussi celui des journalistes et cadres de l'édition. On retrouve là une caractéristique des professions culturelles que de nombreuses études internationales ont contribué à éclairer - et en particulier, parmi elles, des professions artistiques : leur concentration dans les grandes aires métropolitaines ${ }^{7}$.

Graphique 5 - Évolution de la part des professionnels résidant en Île-de-France parmi les actifs en emploi, 1991-2011

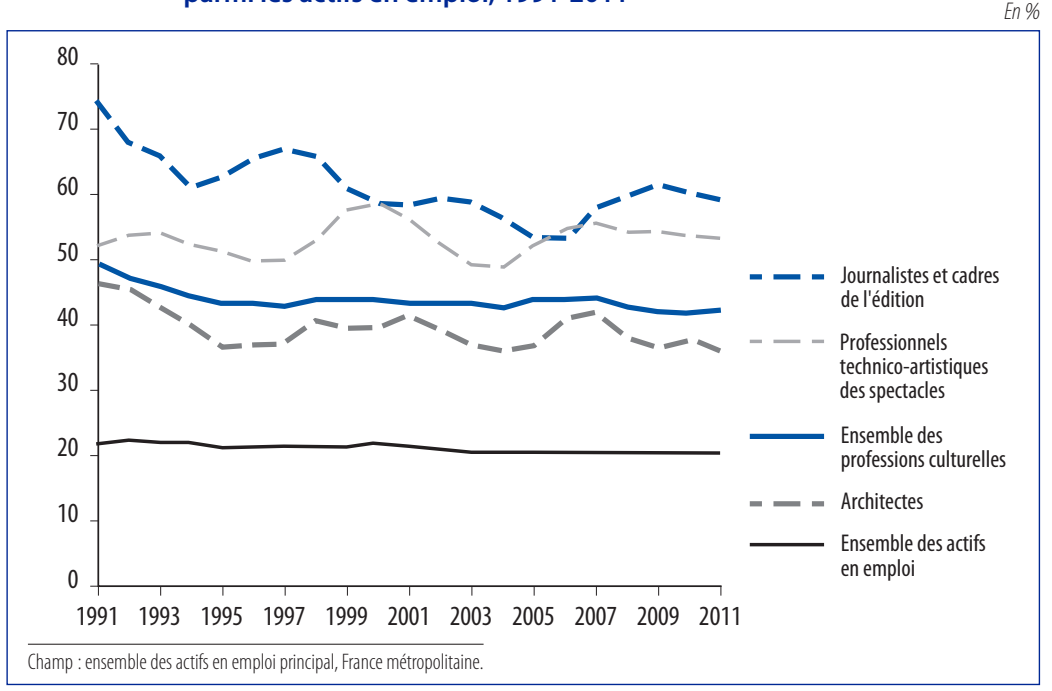

Source: Enquêtes Emploi, Insee/DEPS, Ministère de la Culture et de la Communication, 2014

6. Cédric Delvainguière, François Tugores, Nicolas LAROCHE, Benoît JourdAN, les Dépenses culturelles des collectivités locales en 2010: 7,6 milliards d'euros pour la culture, Paris, Ministère de la Culture et de la Communication, DEPS, coll. « Culture chiffres», 2014-3, 2014.

7. Pierre-Michel MENGER, le Travail créateur. S'accomplir dans l'incertain, Paris, EHESS, Gallimard/Seuil, coll. «Hautes Études», 2009 (chapitre 12). 


\section{Plus souvent nés à l'étranger que les autres}

Les professions culturelles comptent davantage d'actifs nés à l'étranger (14\% en 2011) que la moyenne (11\%) (tableau 3). La proportion d'artistes nés à l'étranger est particulièrement élevée chez les plasticiens (plus de $20 \%$ en 2011) et chez les auteurs littéraires et traducteurs (près de $40 \%$ ). Cette tendance s'est intensifiée au cours des vingt dernières années : la part des artistes plasticiens nés à l'étranger a ainsi crû de 4 points depuis le début des années 1990, celle des auteurs littéraires et traducteurs de 8 points, alors que dans le même temps, dans l'ensemble des professions culturelles et, plus largement, des actifs en emploi, elle n'a que très légèrement progressé. Par rapport aux autres professions, les pays d'origine des professionnels de la culture nés à l'étranger sont plutôt situés en Europe, et moins souvent en Afrique.

Tableau 3 - Pays de naissance des actifs en emploi en 1991 et 2011

\begin{tabular}{|c|c|c|c|c|}
\hline & \multicolumn{2}{|c|}{ Pays étranger } & \multicolumn{2}{|c|}{$\begin{array}{c}\text { dont } \\
\text { pays européen (UE27) }\end{array}$} \\
\hline & 1991 & 2011 & 1991 & 2011 \\
\hline Professions des arts visuels et métiers d'art & 13 & 13 & 47 & 46 \\
\hline Artistes plasticiens & 18 & 22 & 61 & 46 \\
\hline Photographes & 8 & 6 & 37 & 51 \\
\hline Professions des arts graphiques, de la mode et de la décoration & 12 & 13 & 33 & 44 \\
\hline Métiers d'art & 15 & 6 & 53 & 55 \\
\hline Professions des spectacles & 11 & 12 & 46 & 44 \\
\hline Artistes des spectacles & 15 & 15 & 62 & 54 \\
\hline Professionnels technico-artistiques des spectacles & 8 & 11 & 22 & 35 \\
\hline Professions littéraires & 18 & 19 & 44 & 58 \\
\hline Journalistes et cadres de l'édition & 12 & 10 & 30 & 44 \\
\hline Auteurs littéraires et traducteurs & 31 & 39 & 57 & 67 \\
\hline $\begin{array}{l}\text { Professions de l'archivage, de la conservation } \\
\text { et de la documentation }\end{array}$ & 9 & 12 & 43 & 38 \\
\hline Architectes & 12 & 16 & 25 & 35 \\
\hline Professeurs d'art & 9 & 13 & 31 & 40 \\
\hline Ensemble des professions culturelles & 13 & 14 & 43 & 46 \\
\hline Ensemble des actifs en emploi & 9 & 11 & 42 & 34 \\
\hline
\end{tabular}

Source: Enquêtes Emploi, Insee/DEPS, Ministère de la Culture et de la Communication, 2014

\section{La constance d'une origine sociale favorisée}

Au début des années 1990, $45 \%$ des actifs des professions culturelles avaient un père cadre ${ }^{8}$ contre moins de $20 \%$ de l'ensemble des actifs en emploi. Vingt ans plus tard, cette caractéristique forte des métiers artistiques et culturels demeure : un actif

8. Cadre, c'est-à-dire, ici, appartenant aux catégories socioprofessionnelles « cadres et professions intellectuelles supérieures » (médecin, avocat, ingénieur, cadre d'entreprise, personnel de catégorie A de la fonction publique, etc.) ou aux « professions intermédiaires » (technicien, contremaître, instituteur, infirmier, personnel de catégorie B de la fonction publique, etc.). 
des professions culturelles est deux fois plus souvent enfant de cadre qu'un autre actif (tableau 2).

Cette spécificité concerne l'ensemble des professions culturelles, que l'emploi exercé soit lui-même qualifié au niveau cadre ou non. Ainsi, les actifs des métiers d'art travaillant sous le statut d'artisan ou d'ouvrier d'art, et ceux exerçant un métier technique non-cadre du spectacle sont eux aussi deux fois plus souvent enfants de cadre que l'ensemble des actifs non-cadres au niveau national (un tiers contre seulement $15 \%$ des non-cadres, toutes professions confondues).

Dans certaines professions culturelles, cette appartenance familiale aux classes sociales supérieures s'est particulièrement renforcée au cours de la période : c'est le cas, par exemple, des professionnels techniques des spectacles (+ 10 points en vingt ans) et surtout des métiers d'art (+ 12 points) qui étaient beaucoup plus souvent, au début des années 1990, issus des classes moyennes et de familles d'artisans ou d'ouvriers.

\section{Des professionnels de plus en plus diplômés}

En lien avec leur origine sociale élevée, mais aussi avec la nature et le niveau des compétences nécessaires à l'exercice des métiers concernés, les actifs des professions culturelles se caractérisent de longue date par un niveau de diplôme lui aussi élevé : au début des années 1990, un professionnel sur quatre était titulaire d'un diplôme de niveau bac +3 (ou plus), contre un actif sur dix dans l'ensemble des professions (tableau 4).

Au cours des vingt années suivantes, le niveau de diplôme de l'ensemble des actifs, toutes professions confondues, s'est continûment élevé ${ }^{9}$, mais ce mouvement a été plus marqué encore dans les professions culturelles : $41 \%$ des actifs de ces professions en 2011 sont titulaires d'un diplôme de niveau égal ou supérieur à bac +3 , contre $19 \%$ seulement de l'ensemble des actifs en emploi (tableau 4). Ce surclassement en termes de niveau de diplôme rapproche fortement les professions culturelles dans leur ensemble de la population des cadres supérieurs et intermédiaires (dont $39 \%$ sont titulaires d'un diplôme de niveau équivalent ou supérieur à bac +3 en 2011).

En termes d'évolution, deux cas de figure prévalent dans les professions culturelles : certaines d'entre elles étaient déjà, au début des années 1990, occupées par des personnes fortement diplômées et elles le sont encore davantage aujourd'hui ; les autres ont vu se développer la part des diplômés de l'enseignement supérieur, tout en conservant une part importante de titulaires de diplômes de la voie professionnelle (CAP OU BEP).

Dans la première catégorie se trouvent les architectes, les professionnels de l'archivage, de la conservation et de la documentation, les journalistes et cadres de l'édition, ainsi que les auteurs littéraires et les traducteurs : en 2011, les diplômés de niveau égal ou supérieur à bac +3 y représentent plus des deux tiers des effectifs, soit 10 à 20 points de plus en vingt ans. Les artistes plasticiens et les professeurs d'art se rattachent également à cette catégorie, mais dans une moindre mesure.

En revanche, les professionnels techniques des spectacles, ceux des arts graphiques, de la mode et de la décoration, les photographes, les professionnels des métiers d'art - métiers reposant sur des compétences et des savoir-faire techniques

9. Jean-Pierre Dalous, Laurence DAuphin, Martine JelJoul, Nadine LAïB, Béatrice Le Rhun, Jacqueline PERRIN-HAYNES, Isabelle ROBERT-BoBÉE, Scolarisation et origines sociales depuis les années 1980 : progrès et limites, Paris, Insee, coll. «Insee Références. 30 ans de vie économique et sociale », 2014. 
Tableau 4 - Caractéristiques de formation initiale et de conditions d'emploi des actifs en emploi en 1991 et 2011

\begin{tabular}{|c|c|c|c|c|c|c|c|c|c|c|}
\hline & \multicolumn{4}{|c|}{ Formation initiale } & \multicolumn{6}{|c|}{ Emploi } \\
\hline & \multirow{2}{*}{\multicolumn{2}{|c|}{$\mathrm{Bac}+3 \mathrm{ou}+$}} & \multirow{2}{*}{\multicolumn{2}{|c|}{ CAP-BEP }} & \multirow{2}{*}{\multicolumn{2}{|c|}{ Non-salariés }} & \multicolumn{4}{|c|}{ Salariés } \\
\hline & & & & & & & \multicolumn{2}{|c|}{ Contrats courts } & \multicolumn{2}{|c|}{ Temps partie } \\
\hline & 1991 & 2011 & 1991 & 2011 & 1991 & 2011 & 1991 & 2011 & 1991 & 2011 \\
\hline $\begin{array}{l}\text { Professions des arts visuels } \\
\text { et métiers d'art }\end{array}$ & 9 & 26 & 25 & 14 & 48 & 51 & 7 & 14 & 8 & 14 \\
\hline Artistes plasticiens & 24 & 39 & 13 & 10 & 94 & 79 & & & & \\
\hline Photographes & 6 & 22 & 28 & 17 & 54 & 75 & & & & \\
\hline $\begin{array}{l}\text { Professions des arts graphiques, } \\
\text { de la mode et de la décoration }\end{array}$ & 8 & 28 & 21 & 9 & 29 & 40 & 9 & 14 & 9 & 13 \\
\hline Métiers d'art & 2 & 13 & 37 & 33 & 43 & 53 & 3 & 14 & 5 & 8 \\
\hline Professions des spectacles & 12 & 32 & 18 & 10 & 29 & 15 & 37 & 53 & 31 & 28 \\
\hline Artistes des spectacles & 12 & 31 & 11 & 8 & 29 & 17 & 41 & 74 & 45 & 51 \\
\hline $\begin{array}{l}\text { Professionnels technico-artistiques } \\
\text { des spectacles }\end{array}$ & 12 & 33 & 24 & 11 & 30 & 14 & 34 & 40 & 20 & 15 \\
\hline Professions littéraires & 45 & 66 & 2 & 2 & 29 & 28 & 9 & 20 & 15 & 24 \\
\hline Journalistes et cadres de l'édition & 44 & 67 & 3 & 2 & 15 & 12 & 8 & 18 & 12 & 22 \\
\hline Auteurs littéraires et traducteurs & 50 & 65 & 1 & 2 & 62 & 67 & & & & \\
\hline $\begin{array}{l}\text { Professions de l'archivage, } \\
\text { de la conservation } \\
\text { et de la documentation }\end{array}$ & 47 & 68 & 9 & 6 & 0 & 0 & 6 & 9 & 28 & 19 \\
\hline Architectes & 70 & 78 & 3 & 3 & 65 & 56 & 10 & 10 & 8 & 11 \\
\hline Professeurs d'art & 30 & 35 & 8 & 3 & 26 & 19 & 5 & 26 & 39 & 66 \\
\hline $\begin{array}{l}\text { Ensemble des } \\
\text { professions culturelles }\end{array}$ & 26 & 41 & 15 & 9 & 36 & 33 & 15 & 30 & 21 & 26 \\
\hline Ensemble des actifs en emploi & 9 & 19 & 29 & 25 & 15 & 12 & 7 & 13 & 12 & 19 \\
\hline
\end{tabular}

Source: Enquêtes Emploi, Insee/DEPS, Ministère de la Culture et de la Communication, 2014

très spécifiques et hautement qualifiés - relèvent de la seconde catégorie: une proportion de titulaires de CAP et BEP plus élevée qu'ailleurs, mais qui s'est néanmoins réduite au cours de la période au profit des diplômés de l'enseignement supérieur.

\section{L'indépendance est toujours trois fois plus fréquente qu'ailleurs}

Le statut d'indépendant demeure une caractéristique forte de l'emploi dans les professions culturelles : un tiers des actifs exercent en effet leur métier dans un cadre non-salarié en 2011 , soit près de trois fois plus que dans la population active dans son ensemble (tableau 4). Le métier d'artiste plasticien demeure, comme vingt ans plus tôt, le métier le plus massivement exercé sous ce statut ( $79 \%$ de non-salariés en 
2011), même si cette caractéristique dominante a reculé par rapport au début des années 1990 (graphique 6). Il en va de même pour le métier d'architecte où l'exercice non-salarié domine encore, mais décroît, passant de $65 \%$ à $56 \%$ des effectifs en vingt ans. La progression du salariat dans ce métier traditionnellement indépendant s'est effectuée à la faveur d'un double mouvement d'expansion globale des effectifs (+ $45 \%$ ) et d'ouverture aux femmes, dont la part a doublé - celles-ci adoptant beaucoup plus que les hommes l'exercice salarié de la profession (parmi les $44 \%$ d'architectes salariés en 2011 , on compte $67 \%$ de femmes pour $33 \%$ d'hommes).

Les autres professions du champ étudié sont des professions où, à l'inverse, le salariat domine de longue date et reste majoritaire en 2011. Les professeurs d'art, les journalistes et cadres de l'édition ainsi que les artistes et professionnels techniques des spectacles étaient majoritairement salariés en 1991 et le sont aujourd'hui davantage : le non-salariat ne concerne plus que $10 \%$ à $20 \%$ des effectifs. Parmi les professions à dominante salariée, seules les professions des arts graphiques, de la mode et de la décoration ont vu progresser nettement la part des non-salariés au cours de la période (+ 11 points). L'exercice indépendant y concerne désormais $40 \%$ des actifs.

\section{Graphique 6 - Évolution de la part des non-salariés parmi les actifs en emploi,} 1991-2011

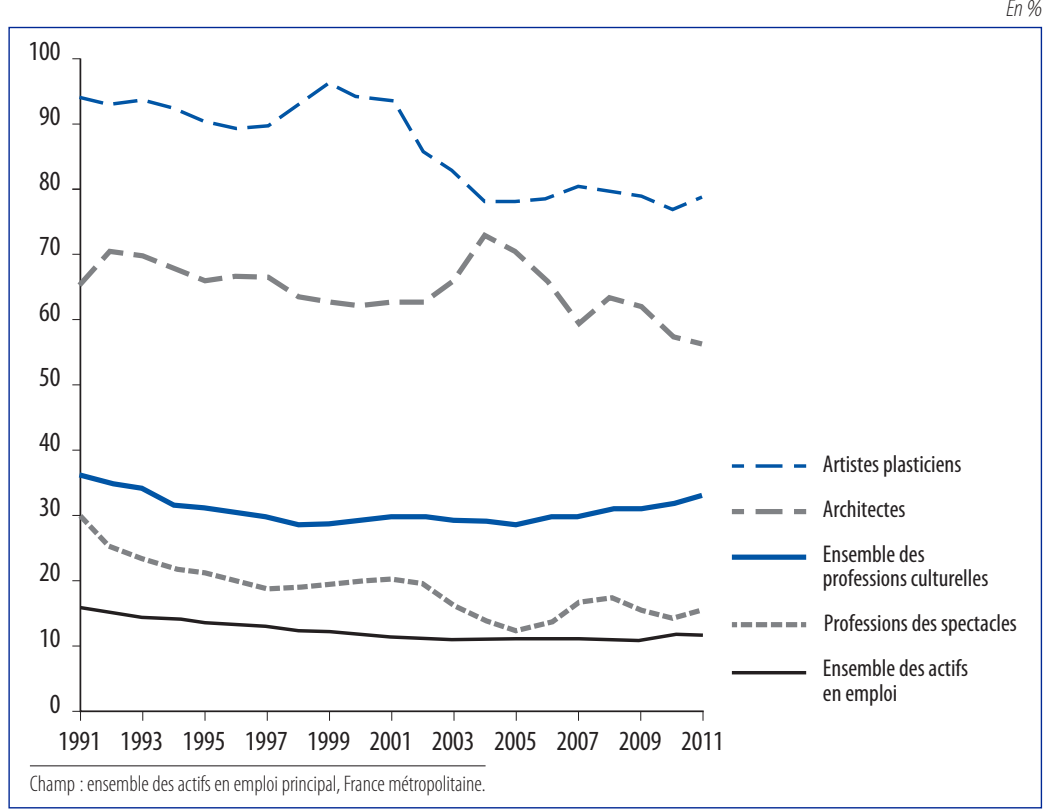

Source: Enquêtes Emploi, Insee/DEPS, Ministère de la Culture et de la Communication, 2014 


\section{Au sein du salariat, les contrats courts et le travail à temps partiel continuent de se développer}

Dans les professions culturelles comme dans l'ensemble de l'économie, la part des contrats courts ${ }^{10}$ au sein du salariat a doublé au cours des vingt ans étudiés (tableau 4 et graphique 7). Avec la hausse du temps partiel, il s'agit là d'un des indicateurs clés du mouvement de fragmentation et de diversification du salariat observé sur l'ensemble du marché du travail ${ }^{11}$. Dans le salariat culturel, la part des contrats courts était déjà, en 1991, deux fois plus importante que dans l'ensemble de la population salariée : $15 \%$, contre $7 \%$ seulement de l'ensemble des salariés. Elle le demeure donc aujourd'hui, avec $30 \%$ de salariés sur contrats courts. Le travail à temps partiel était lui aussi plus répandu dans le salariat culturel au début des années 1990 et il a fortement progressé en vingt ans, pour concerner un quart ( $26 \%$ ) des salariés de ce champ en 2011.

La progression de la part des contrats courts et du temps partiel ne s'observe cependant pas dans tous les métiers artistiques et culturels. Certains échappent même à ce phénomène.

C'est parmi les salariés des spectacles que ces formes flexibles du salariat sont les plus répandues : $74 \%$ des artistes et $40 \%$ des professionnels techniques des spectacles travaillent sous contrat court, dont en grande majorité deS CDD dits « d'usage constant », caractéristiques du régime de l'intermittence (encadré, § 5, p. 22 ). Plus de la moitié des artistes se déclarent à temps partiel, tandis que c'est de moins en moins souvent le cas des salariés techniciens du spectacle, qui s'éloignent donc radicalement des

\section{Graphique 7 - Évolution de la part des salariés en contrats courts parmi les salariés en emploi, 1991-2011}

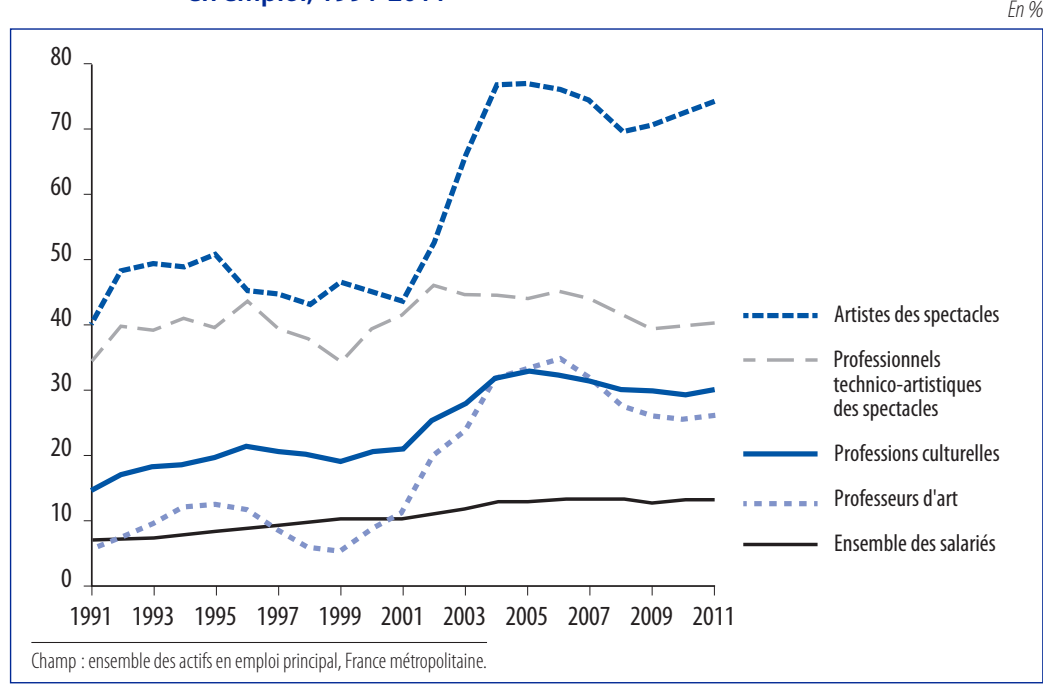

Source: Enquêtes Emploi, Insee/DEPS, Ministère de la Culture et de la Communication, 2014

10. Contrats à durée déterminée (CDD) et autres contrats temporaires : contrats aidés, vacations, missions d'intérim...

11. Alain SuPIOT, Au-delà de l'emploi : transformations du travail et devenir du droit du travail en Europe : rapport pour la Commission des Communautés européennes, Paris, Flammarion, 1999. 


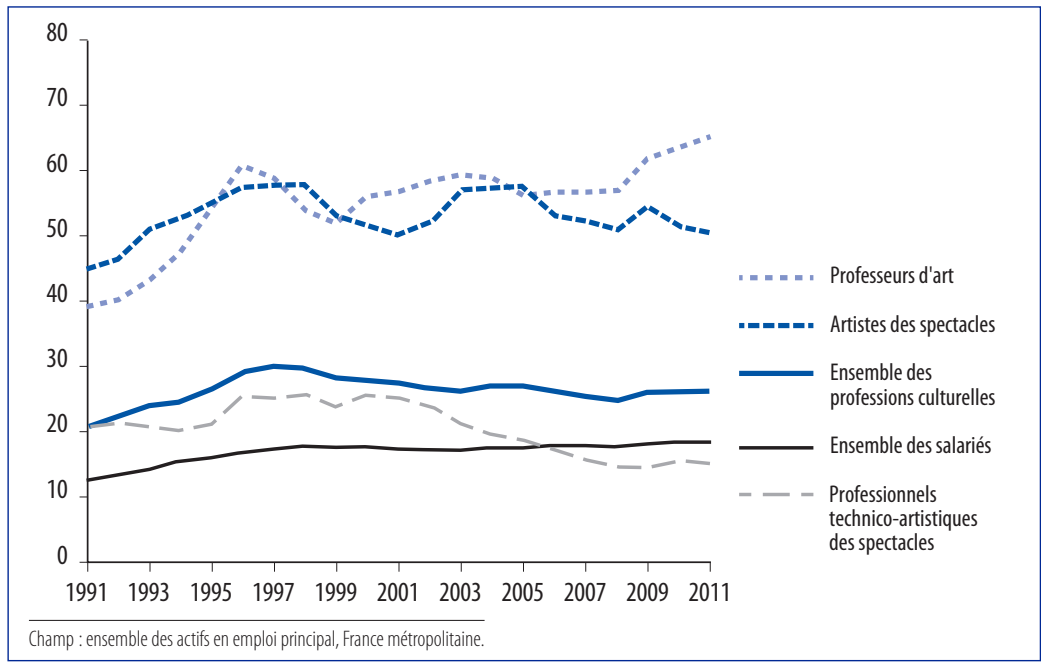

Source : Enquêtes Emploi, Insee/DEPS, Ministère de la Culture et de la Communication, 2014

artistes du spectacle à cet égard (tableau 4, graphiques 7 et 8). Le développement marqué de ces deux caractéristiques d'emploi parmi les professeurs d'art ( +21 points pour les contrats courts et +27 points pour le temps partiel) rapproche aujourd'hui fortement ces derniers des artistes du spectacle. Journalistes et cadres de l'édition sont eux aussi concernés par ce double mouvement de fragmentation de l'emploi, bien que dans une moindre mesure.

D'autres professions majoritairement exercées dans le cadre du salariat sont au contraire peu concernées par le développement de ces formes flexibles d'emploi, comme les professions des arts graphiques, de la mode et de la décoration, celles de l'archivage, de la conservation et de la documentation, ou encore les ouvriers d'art. Entre $80 \%$ et $85 \%$ des salariés de ces métiers exercent en effet leur activité professionnelle en CDI et à temps complet. II en va de même des architectes salariés - minoritaires au sein de cette profession - qui se rapprochent eux aussi de ce profil plus classique où domine l'emploi permanent à temps plein : $11 \%$ seulement des architectes salariés travaillent à temps partiel.

\section{Un temps partiel plus court et plus souvent contraint}

Plus répandu dans le champ culturel, le salariat à temps partiel présente certaines spécificités. Il touche par exemple les hommes et les femmes de façon assez similaire, alors qu'il est avant tout une caractéristique de l'emploi féminin dans l'ensemble de la population salariée ${ }^{12}$. 
Autre caractéristique du temps partiel dans les professions culturelles : la quotité de temps partiel est souvent plus faible qu'ailleurs (tableau 5). En 2011, dans les professions culturelles, $58 \%$ des salariés à temps partiel travaillent moins d'un mitemps, contre $40 \%$ de l'ensemble des salariés à temps partiel. Cette caractéristique est notamment très marquée pour les salariés des spectacles, où la notion de temps partiel renvoie davantage à un temps de travail extrêmement fractionné sur de multiples contrats très courts qu'à l'exercice classique du travail à temps partiel chez les salariés (absence de travail d'une demi-journée ou d'une journée par semaine).

Tableau 5 - Temps partiel et sous-emploi parmi les salariés en emploi en 2011

\begin{tabular}{|c|c|c|c|c|c|c|c|c|c|c|}
\hline & \multicolumn{10}{|c|}{ Emploi salarié à temps partiel } \\
\hline & \multirow[b]{2}{*}{$\%$} & \multicolumn{3}{|c|}{$\begin{array}{c}\text { Quotité } \\
\text { du temps partiel }\end{array}$} & \multicolumn{5}{|c|}{ Motif principal du temps partiel } & \multirow{2}{*}{ 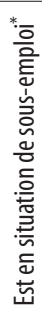 } \\
\hline & & 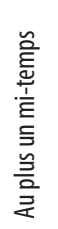 & 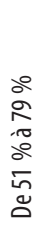 & $\begin{array}{l}+ \\
z \\
\vdots \\
\circ \\
\infty\end{array}$ & 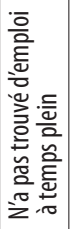 & 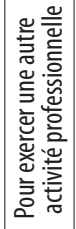 & 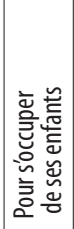 & 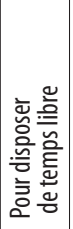 & 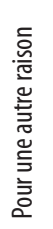 & \\
\hline \multirow{4}{*}{$\begin{array}{l}\text { Salariés des arts visuels } \\
\text { et métiers d'art } \\
\text { Artistes plasticiens } \\
\text { Photographes } \\
\text { Professions des arts graphiques, } \\
\quad \text { de la mode et de la décoration } \\
\text { Ouvriers d'art }\end{array}$} & 14 & 45 & 20 & 35 & 26 & 20 & 24 & 19 & 10 & 19 \\
\hline & & & & & & & & & & \\
\hline & 13 & 42 & 19 & 39 & 21 & 23 & 26 & 20 & 10 & 16 \\
\hline & 8 & & & & & & & & & \\
\hline \multirow{3}{*}{$\begin{array}{l}\text { Salariés des spectacles } \\
\text { Artistes des spectacles } \\
\text { Professionnels techniques } \\
\text { des spectacles }\end{array}$} & 28 & 64 & 24 & 11 & 52 & 12 & 7 & 6 & 24 & 49 \\
\hline & 51 & 69 & 22 & 9 & 57 & 10 & 3 & 7 & 23 & 53 \\
\hline & & 56 & 29 & 15 & 43 & 14 & 14 & 4 & 25 & 42 \\
\hline Salariés des professions littéraires & 24 & 46 & 32 & 22 & 31 & 21 & 23 & 16 & 9 & 28 \\
\hline \multirow{2}{*}{$\begin{array}{l}\text { Journalistes et cadres de l'édition } \\
\text { Auteurs littéraires et traducteurs }\end{array}$} & 22 & 40 & 34 & 26 & 32 & 24 & 20 & 13 & 11 & 25 \\
\hline & & & & & & & & & & \\
\hline $\begin{array}{l}\text { Salariés de l'archivage, } \\
\text { de la conservation } \\
\text { et de la documentation }\end{array}$ & 19 & 31 & 22 & 48 & 11 & 9 & 35 & 21 & 23 & 12 \\
\hline Architectes salariés & 11 & & & & & & & & & \\
\hline Professeurs d'art salariés & 66 & 71 & 24 & 5 & 44 & 29 & 12 & 7 & 8 & 39 \\
\hline $\begin{array}{l}\text { Salariés des professions } \\
\text { culturelles }\end{array}$ & 26 & 58 & 24 & 19 & 40 & 18 & 17 & 11 & 15 & 37 \\
\hline Ensemble des salariés & 19 & 40 & 27 & 33 & 32 & 9 & 29 & 15 & 15 & 28 \\
\hline \multicolumn{11}{|c|}{ 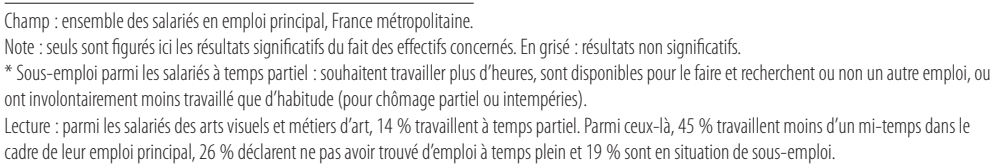 } \\
\hline
\end{tabular}


Interrogés sur la raison pour laquelle ils exercent à temps partiel, $18 \%$ des salariés des professions culturelles expliquent exercer en parallèle une autre activité professionnelle (contre $9 \%$ de l'ensemble des salariés à temps partiel). Plus d'un quart des professeurs d'art, par exemple, sont concernés et associent l'enseignement à l'exercice d'un métier de la création ou de l'interprétation artistiques (artiste des spectacles, artiste plasticien, etc.).

Surtout, le temps partiel dans le salariat culturel est rarement choisi : $40 \%$ des salariés déclarent en effet travailler à temps partiel faute d'avoir trouvé un emploi à temps plein, contre $32 \%$ de l'ensemble des salariés à temps partiel (tableau 5).

Ces caractéristiques traduisent la fréquence des situations de sous-emploi ${ }^{13}$ dans les métiers culturels : parmi les salariés à temps partiel, $37 \%$ des salariés exerçant à temps partiel au titre de leur emploi principal une profession culturelle sont sousemployés, contre $28 \%$ de l'ensemble des salariés à temps partiel.

\section{Une plus grande plasticité des jours et horaires de travail}

Dans l'ensemble de la population active, les rythmes et modes d'organisation du travail ont subi d'importantes évolutions au cours de ces dernières années : davantage de travail le week-end, développement de la variabilité des jours et des horaires de travail, plus grande diversité des volumes hebdomadaires d'heures travaillées, notamment $^{14}$.

À cet égard, les professions culturelles présentent aujourd'hui des modalités d'organisation encore plus atypiques : en $2011^{15}$, les actifs des professions culturelles déclarent en effet travailler en horaires variables d'une semaine à l'autre beaucoup plus souvent que les autres professions ( $46 \%$, contre $26 \%$ dans l'ensemble des actifs en emploi) ; un quart d'entre eux travaillent habituellement le soir contre $17 \%$ seulement des actifs toutes professions confondues (tableau 6).

Les métiers culturels se répartissent de ce point de vue en quatre grands groupes.

Le premier est composé des métiers des spectacles et de la communication dont l'exercice implique une grande souplesse d'organisation. Les professionnels techniques des spectacles, et plus encore les artistes des spectacles sont ainsi nombreux à travailler en horaires variables ( $57 \%) ; 41 \%$ travaillent habituellement le soir, $16 \%$ la nuit et $32 \%$ le dimanche, du fait des jours et horaires des spectacles et des tournages. Les journalistes et cadres de l'édition, chargés du suivi en continu de l'information dans les rédactions, relèvent également de cette catégorie : plus de la moitié des actifs travaillent en horaires variables et un quart d'entre eux travaillent habituellement le soir et le week-end.

Le deuxième groupe, essentiellement composé de non-salariés - artistes plasticiens, photographes, architectes, artisans d'art, auteurs littéraires et traducteurs témoigne lui aussi d'une organisation atypique du temps de travail, partageant cette caractéristique avec les autres non-salariés (médecins, avocats, mais aussi artisans, commerçants, etc.) qui déclarent souvent des horaires variables d'une semaine sur l'autre $(50 \%)$, et qui travaillent habituellement le soir après 20 heures $(21 \%)$ et le dimanche (27\%).

13. En situation de sous-emploi : salariés qui souhaitent travailler plus d'heures et se déclarent disponibles pour le faire, ou bien ont involontairement moins travaillé que d'habitude pour cause de chômage partiel ou d'intempéries.

14. Voir par exemple, Olivia SAUTORY, Sandra ZILLONIZ, «Les rythmes de travail en 2010 », Document d'études n 180, DARES, avril 2014.

15. Les questions relatives aux jours et horaires de travail ont évolué dans l'enquête Emploi au fil du temps. Il est donc malheureusement impossible de raisonner ici en termes d'évolution. 


\begin{tabular}{|c|c|c|c|c|}
\hline & \multirow{2}{*}{$\begin{array}{c}\text { En horaires } \\
\text { variables } \\
\text { d'une semaine } \\
\text { sur l'autre }\end{array}$} & \multicolumn{3}{|c|}{ Travaille habituellement } \\
\hline & & $\begin{array}{l}\text { le soir } \\
\text { (entre } 20 \mathrm{~h} \\
\text { et minuit) }\end{array}$ & $\begin{array}{c}\text { la nuit } \\
\text { (entre minuit } \\
\text { et } 5 \mathrm{~h} \text { ) }\end{array}$ & le dimanche \\
\hline $\begin{array}{l}\text { Professions des arts visuels } \\
\text { et métiers d'art } \\
\text { Artistes plasticiens } \\
\text { Photographes } \\
\text { Professions des arts graphiques, } \\
\quad \text { de la mode et de la décoration } \\
\text { Métiers d'art }\end{array}$ & $\begin{array}{l}44 \\
71 \\
66 \\
37 \\
33\end{array}$ & $\begin{array}{l}16 \\
26 \\
26 \\
13 \\
12\end{array}$ & $\begin{array}{c}4 \\
4 \\
12 \\
\\
2 \\
4\end{array}$ & $\begin{array}{c}12 \\
26 \\
24 \\
\\
6 \\
10\end{array}$ \\
\hline $\begin{array}{l}\text { Professions des spectacles } \\
\text { Artistes des spectacles } \\
\text { Professionnels techniques } \\
\text { des spectacles }\end{array}$ & $\begin{array}{l}57 \\
73 \\
48\end{array}$ & $\begin{array}{l}41 \\
56 \\
32\end{array}$ & $\begin{array}{l}16 \\
19 \\
14\end{array}$ & $\begin{array}{l}32 \\
46\end{array}$ \\
\hline $\begin{array}{l}\text { Professions littéraires } \\
\text { Journalistes et cadres de l'édition } \\
\text { Auteurs littéraires et traducteurs }\end{array}$ & $\begin{array}{l}57 \\
53 \\
67\end{array}$ & $\begin{array}{l}23 \\
24 \\
20\end{array}$ & $\begin{array}{l}5 \\
5 \\
6\end{array}$ & $\begin{array}{l}21 \\
24 \\
12\end{array}$ \\
\hline $\begin{array}{l}\text { Professions de l'archivage, } \\
\text { de la conservation } \\
\text { et de la documentation }\end{array}$ & 18 & 8 & 0 & 2 \\
\hline Architectes & 33 & 13 & 0 & 2 \\
\hline Professeurs d'art & 18 & 36 & 1 & 7 \\
\hline $\begin{array}{l}\text { Professions culturelles } \\
\text { dont : parmi les salariés } \\
\quad \text { parmi les non-salariés }\end{array}$ & $\begin{array}{l}46 \\
35 \\
68\end{array}$ & $\begin{array}{l}25 \\
24 \\
28\end{array}$ & $\begin{array}{l}7 \\
7 \\
7\end{array}$ & $\begin{array}{l}17 \\
16 \\
20\end{array}$ \\
\hline $\begin{array}{l}\text { Ensemble des actifs en emploi } \\
\text { dont : parmi les salariés } \\
\text { parmi les non-salariés }\end{array}$ & $\begin{array}{l}26 \\
22 \\
50\end{array}$ & $\begin{array}{l}17 \\
16 \\
21\end{array}$ & $\begin{array}{l}7 \\
7 \\
5\end{array}$ & $\begin{array}{l}15 \\
13 \\
27\end{array}$ \\
\hline
\end{tabular}

Source : Enquêtes Emploi, Insee/DEPs, Ministère de la Culture et de la Communication, 2014

Le troisième groupe est constitué de métiers qui s'inscrivent dans le cadre classique de l'organisation du travail salarié, avec des horaires le plus souvent réguliers d'une semaine sur l'autre, concentrés sur la journée et en semaine: professions des arts graphiques, de la mode et de la décoration, ouvriers d'art, professions de l'archivage, de la conservation et de la documentation.

Le dernier groupe, enfin, composé des seuls professeurs d'art, présente un profil hybride, à mi-chemin entre les salariés à horaires classiques précédents et les salariés dont le temps de travail est le plus malléable: plus d'un sur trois déclare travailler habituellement après 20 heures. II peut s'agir, par exemple, de cours de théâtre, d'arts plastiques ou de musique dispensés en soirée auprès de pratiquants amateurs (écoles de musique, associations de quartier, etc.). Ce travail du soir les rapproche des autres enseignants ( $46 \%$ de ceux du secondaire et du supérieur travaillent habituellement le soir) pour lesquels cette modalité correspond sans doute davantage au travail de préparation des cours et de correction des devoirs. 


\section{Encadré}

\section{Mesurer l'emploi culturel}

\section{1 - Plusieurs approches possibles}

On peut distinguer deux façons d'aborder l'emploi culturel.

La première consiste à décrire les professions définies comme culturelles, et correspond à l'angle adopté dans la présente étude. On veut en effet y décrire les caractéristiques sociodémographiques typiques des professionnels de la culture, et les conditions de travail propres à ces professions. Les professions culturelles analysées ici sont définies à partir de la nomenclature de 2003 des professions et catégories socioprofessionnelles (PCS).

La seconde détaille les effectifs par secteurs d'activité culturels, effectifs dont les professions peuvent être de nature culturelle ou non (technique, administrative etc.). C'est l'approche qui est par exemple utilisée pour estimer la part de la culture dans l'économie ${ }^{16}$. Les secteurs culturels sont définis par référence à la nomenclature d'activités française (NAF).

Les deux approches se recoupent partiellement (graphique A). En 2011, selon l'enquête Emploi, 573000 personnes exercent une profession culturelle au titre de l'emploi principal, soit 2,2 \% de l'emploi total, et près de 680000 travaillent dans un secteur culturel (2,6\% du total) (tableau A). Parmi elles, 342700 exercent une profession culturelle dans un secteur culturel (par exemple, en tant que graphiste dans une agence publicitaire ou qu'artiste dans une compagnie théâtrale), 230300 exercent une profession culturelle dans un secteur non culturel (par exemple, en tant que designer dans l'industrie). La moitié des personnes en emploi dans les secteurs culturels (337 000) exercent une profession non culturelle (en occupant par exemple un poste administratif dans une compagnie de spectacle vivant ou dans un cabinet d'architecte).

\section{Graphique A - L'emploi culturel par profession et par secteur d'activité en 2011}

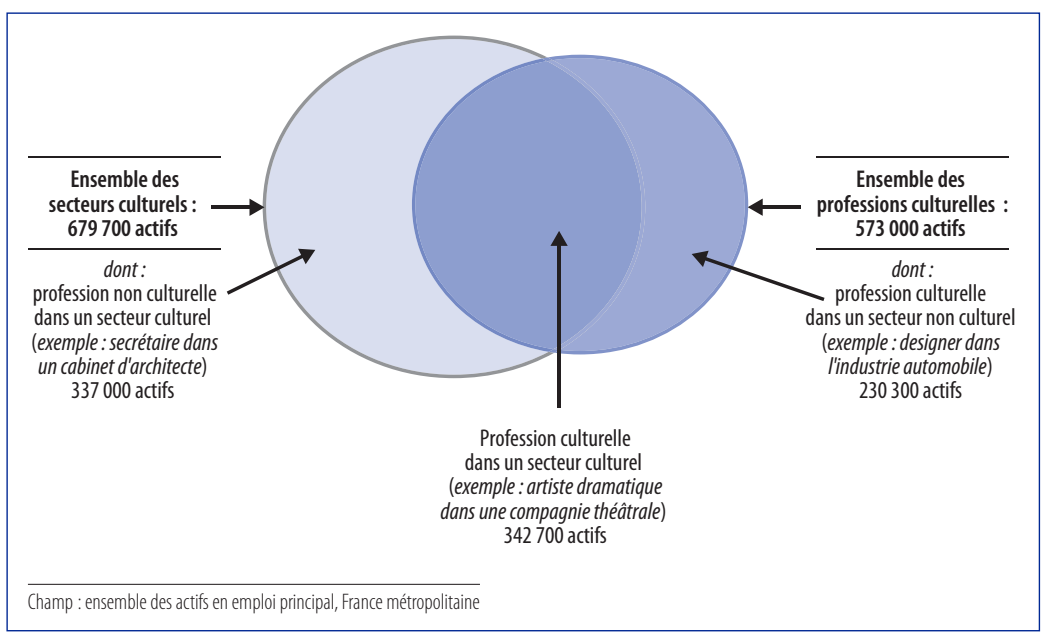

Source: Enquêtes Emploi, Insee/DEPS, Ministère de la Culture et de la Communication, 2014

16. Yves Jauneau, Xavier Niel, le Poids économique direct de la culture en 2013, Paris, Ministère de la culture et de la communication, DEPS, coll. «Culture chiffres », 2014-5, 2014. 
Tableau A - Effectifs en emploi dans les professions culturelles et dans les secteurs culturels en 2011

\begin{tabular}{|lcc|}
\hline Professions & Effectifs & $\begin{array}{c}\text { dont } \% \\
\text { dans un secteur culturel }\end{array}$ \\
Professions des arts visuels et métiers d'art & 194300 & 50 \\
Professions des spectacles & 166000 & 69 \\
Professions littéraires & 88600 & 80 \\
Professions de l'archivage, de la conservation & & 24 \\
$\quad$ et de la documentation & 25900 & 75 \\
Architectes & 51700 & 32 \\
Professeurs d'art & 46500 & 60 \\
\hline Professions culturelles & 573000 & 2,6 \\
\hline Ensemble des actifs en emploi & 25741600 & dont $\%$ \\
\hline Secteurs d'activité & & 80 \\
Arts visuels & Effectifs & 72 \\
Spectacle vivant & & 62 \\
Audiovisuel/multimédia & 95100 & 36 \\
Livre, presse & 104900 & 14 \\
Patrimoine & 101700 & 59 \\
Architecture & 152300 & 68 \\
Enseignement culturel & 50000 & 14 \\
Publicité & 69600 & 50 \\
\hline Secteurs d'activité culturels & 18500 & 2,2 \\
\hline Ensemble des actifs en emploi & 87600 & \\
\hline Champ : ensemble des actifs en emploi principal, France métropolitaine. & & exerçant une profession culturelle \\
\hline
\end{tabular}

Source : Enquêtes Emploi, Insee/DEPS, Ministère de la Culture et de la Communication, 2014

\section{2 - Les enquêtes Emploi de l'Insee}

Les statistiques présentées ici sont tirées des enquêtes Emploi de l'Insee. Ces enquêtes interrogent un échantillon d'individus âgés de 15 ans ou plus résidant en France métropolitaine, tant sur l'emploi que sur le chômage, la formation, l'origine sociale ou encore la situation professionnelle. Avant 2003, ces enquêtes interrogeaient environ 70000 individus chaque année en mars. Depuis 2003, la collecte est réalisée en continu, toutes les semaines de chaque trimestre et interroge désormais, chaque trimestre, 108000 individus. Un même individu est interrogé à six reprises (six trimestres consécutifs) par un enquêteur de l'Insee, en face à face pour la première et la dernière interrogation, par téléphone pour les enquêtes intermédiaires. Le questionnaire, déroulé informatiquement, est doté de nombreux filtres et questions qui permettent de limiter les risques d'erreur de saisie et de corriger les déclarations erronées. Les professions et les secteurs d'activité sont codés de façon particulièrement précise et fiable, à l'aide des nombreuses indications que fournit l'enquêté.

Les données présentées ici sur une année reposent sur la fusion de trois enquêtes consécutives, afin de garantir la robustesse et la fiabilité des résultats sur le champ restreint que constituent les professions culturelles, dans le but de disposer d'effectifs suffisamment importants. L'analyse a ainsi été menée de 1990-1992 (soit une situation « moyenne » en 1991) à 2010-2012 (moyenne en 2011). 


\section{3 - Les professions culturelles dans la nomenclature des professions et catégories socioprofessionnelles (PCS)}

Pour circonscrire le champ des professions culturelles, les codes suivants de la nomenclature des professions et catégories socioprofessionnelles (PCS 2003) sont retenus:
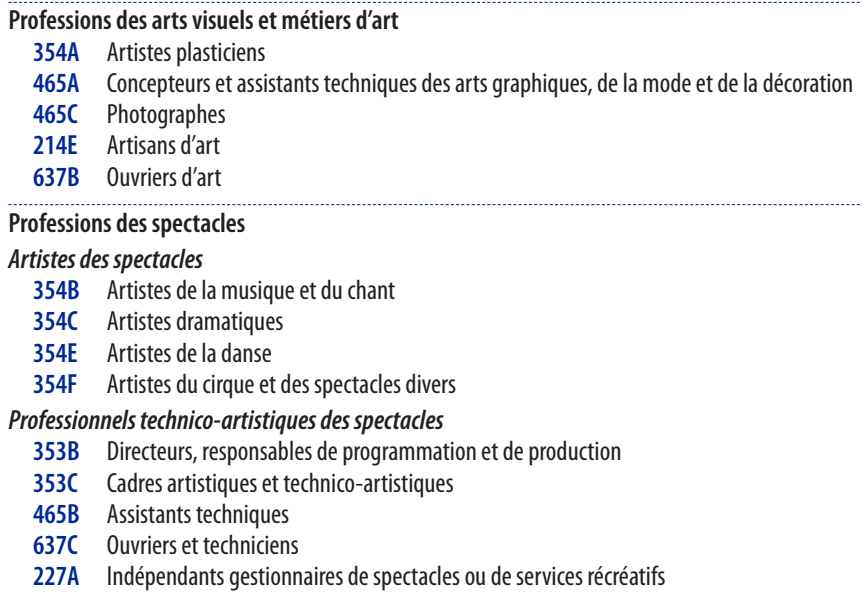

\section{Professions littéraires}

Journalistes et cadres de l'édition

$352 \mathrm{~A}$ Journalistes et rédacteurs en chef

353A Directeurs de journaux, administrateurs de presse, directeurs d'édition

\section{Auteurs littéraires et traducteurs}

352B Auteurs littéraires, scénaristes, dialoguistes

464B Traducteurs et interprètes

Professions de l'archivage, de la conservation et de la documentation

351A Bibliothécaires, archivistes, conservateurs, de la fonction publique

372F Cadres de la documentation, de l'archivage (hors fonction publique)

425A Sous-bibliothécaires, cadres intermédiaires du patrimoine

Architectes
312F Architectes libéraux
382B Architectes salariés

Professeurs d'art (hors établissements scolaires)

354G Professeurs d'art (hors établissements scolaires)

\section{4 - Les secteurs d'activité culturels dans la nomenclature d'activités française (NAF)}

Le contour des secteurs d'activité culturels se fonde sur une définition adoptée de façon conjointe par les différents services statistiques européens de la culture en 2009, sous l'égide d'Eurostat, I'Office statistique de l'Union européenne. Le champ de la culture est ici défini en référence à la nomenclature d'activités française (NAF), par la sélection de 34 codes parmi les 732 qui composent la nomenclature à son niveau le plus désagrégé. Ce contour englobe l'ensemble du périmètre de compétences du ministère de la Culture et de la Communication, ainsi que les agences de publicité, prises en compte dans le champ statistique de la culture pour leur aspect créatif ; les activités des régies publicitaires ne sont en revanche pas prises en compte, car non créatives. Le groupe de travail d'Eurostat a suivi en cela les pratiques de la plupart des autres travaux internationaux du même type. N'ont en revanche pas été retenues par Eurostat les activités industrielles qui ne 
permettent que la reproduction de produits culturels mais qui n'y apportent pas de valeur qu'on aurait pu considérer comme culturelle: l'imprimerie, les équipements et matériaux (depuis la fabrication des optiques jusqu'à celle des peintures ou autres encres ou vernis d'imprimerie) et pour les mêmes raisons la reproduction et la fabrication d'instruments de musique.

Sont retenus les codes suivants:

\section{Arts visuels \\ 9003A Création artistique relevant des arts plastiques \\ 9003B Autre création artistique \\ $7410 Z$ Activités spécialisées de design \\ $7420 Z$ Activités photographiques}

\section{Spectacle vivant}

90012 Arts du spectacle vivant

$9002 Z$ Activités de soutien au spectacle vivant

$9004 Z$ Gestion de salles de spectacles

\footnotetext{
Audiovisuel/multimédia

5911A Production de films et de programmes pour la télévision

5911B Production de films institutionnels et publicitaires

5911C Production de films pour le cinéma

$5912 Z$ Postproduction de films cinématographiques, de vidéo et de programmes de télévision

5913A Distribution de films cinématographiques

5913B Édition et distribution vidéo

$5914 Z$ Projection de films cinématographiques

$5920 Z$ Édition d'enregistrements sonores

$6010 Z$ Édition et diffusion de programmes radio

$6020 \mathrm{~A}$ Édition de chaînes généralistes

6020B Édition de chaînes thématiques

$7722 Z$ Location de cassettes et disques vidéo

$4763 Z$ Commerce de détail d'enregistrements musicaux et vidéo en magasin spécialisé

58212 Édition de jeux électroniques
}

Livre

58112 Édition de livres

47612 Commerce de détail de livres en magasin spécialisé

$7430 Z$ Traduction et interprétation

\section{Presse}

58132 Édition de journaux

$5814 Z$ Édition de revues et périodiques

47622 Commerce de détail de journaux et papeterie en magasin spécialisé

63912 Agences de presse

\section{Patrimoine}

91012 Gestion des bibliothèques et des archives

$9102 Z$ Gestion des musées

$9103 Z$ Gestion des sites et monuments historiques et des attractions touristiques similaires

\section{Architecture}

71112 Activités d'architecture

\section{Enseignement}

$8552 Z$ Enseignement culturel

\section{Agences de publicité}

73112 Activités des agences de publicité 


\section{5 - L'intermittence dans les métiers du spectacle}

L'enquête Emploi mobilisée ici ne permet pas de rendre compte statistiquement de l'intermittence dans les métiers du spectacle, ni selon l'approche par profession, ni selon celle par secteur d'activité ; la nature particulière des contrats de travail sur lesquels s'appuie le dispositif de l'intermittence n'est en effet pas identifiée en tant que telle dans le questionnaire d'enquête.

\section{Le contrat}

Le salariat intermittent repose sur une forme particulière de contrat de travail : le CDD dit « d'usage constant » ou CDDU, qui est devenu progressivement la forme d'emploi la plus courante pour les artistes et techniciens des spectacles. Le recours à ce contrat est encadré juridiquement par le Code du travail qui liste les secteurs d'activité dans lesquels il peut s'appliquer et le type d'emplois concernés (qui doivent être « par nature » temporaires).

Ce cDDu peut être conclu pour une durée extrêmement courte. De ce fait, une partie importante de la population intermittente (variable d'une année à l'autre) effectue un faible volume de travail sous cette forme contractuelle d'emploi. Par exemple, un figurant sur un tournage sera «intermittent » quelques heures dans l'année et peut exercer par ailleurs une tout autre profession, dans un autre secteur que celui du spectacle.

D'autres salariés intermittents, en revanche, effectuent toute leur activité professionnelle dans le spectacle en cumulant des CDDU, parfois avec un grand nombre d'employeurs différents.

\section{Le régime d'assurance chômage}

Le régime actuel d'assurance chômage des intermittents a été créé dans les années 1960, sous forme de deux annexes à la convention de I'Unedic: I'annexe VIII assure le risque de chômage des cadres, techniciens et ouvriers des spectacles travaillant pour un employeur de l'audiovisuel ou du spectacle vivant, tandis que l'annexe $\mathrm{X}$ indemnise les artistes des spectacles, quelle que soit l'activité de l'employeur (que le spectacle soit son activité principale ou non).

Actuellement, tout salarié ayant effectué plus de 507 heures de travail sur dix mois et demi (pour les artistes) ou sur dix mois (pour les techniciens) peut bénéficier de l'indemnisation chômage au titre des annexes VIII et X pour ses périodes non travaillées.

\section{Les sources professionnelles}

Certaines sources repèrent les CDDU : Pôle Emploi et la Caisse des congés spectacles, notamment.

En 2011, Pôle Emploi recense ainsi 254000 salariés ayant travaillé une heure ou plus dans le champ des annexes VIII et X à la convention d'assurance chômage. Ce dénombrement inclut les personnes qui n'ont travaillé qu'un nombre extrêmement réduit d'heures et qui peuvent ne pas exercer leur profession principale dans le secteur culturel.

Dans le même temps, Pôle Emploi dénombre 98700 salariés indemnisés par l'assurance chômage au cours de l'année, au titre de ces deux annexes. S'agissant de personnes qui ont fait la démarche de se faire indemniser pour leur période de chômage, elles ont donc travaillé au moins 507 heures sur l'année dans le champ des annexes VIII et X.

La Caisse des congés spectacles recense, au sein de la population des salariés intermittents ayant obtenu au moins un contrat sous forme de CDDU dans l'année, ceux dont les droits à congés ont été payés, après en avoir fait expressément la demande auprès de la Caisse ; il s'agit donc d'un protocole déclaratif, et non automatique contrairement à Pôle Emploi. Cela suppose non seulement, pour l'intéressé, de disposer d'un bon niveau d'information sur les droits sociaux et procédures réglementaires propres à ces métiers, mais sans doute aussi d'avoir travaillé suffisamment dans l'année pour que le montant des droits à congés payés ne soit pas considéré comme négligeable financièrement. Par voie de conséquence, ceux des salariés en CDDU qui ne sont pas dénombrés par la Caisse des congés spectacles nont donc pas réclamé le versement de leurs droits à congés et 
peuvent être considérés comme des intervenants très occasionnels (par exemple, des figurants pour le tournage d'un film, des musiciens de renfort pour une prestation d'orchestre, etc.). En revanche, ceux qui sont dénombrés peuvent avoir quand même travaillé moins de 507 heures, le versement des congés payés n'étant pas soumis aux mêmes règles que l'indemnisation du chômage au titre des annexes VIII et X.

La Caisse des congés spectacles dénombrait 154000 intermittents en 2009 dans les secteurs du spectacle (derniers chiffres publiés).

\section{À lire aussi}

\section{CC2014-2}

\section{Tendances de l'emploi dans le spectacle}

Marie Gouyon, Frédérique Patureau

Janvier 2014, 8 p.

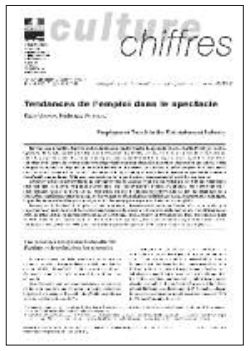

En vingt ans, le nombre d'artistes et de techniciens de l'audiovisuel et du spectacle vivant a doublé. Portée par le développement de l'emploi salarié intermittent, cette croissance des effectifs s'est inscrite dans un marché du travail extrêmement dynamique en termes de créations d'entreprises (tout particulièrement dans le spectacle vivant) et de volume de travail offert. Pour autant, la croissance des effectifs professionnels s'est effectuée à un rythme plus soutenu que celui de l'offre d'emploi, instaurant une dégradation des situations individuelles mesurée par la baisse de près d'un quart du volume annuel de travail et des rémunérations au cours des années 1990. Après quelques années de stabilisation, la reprise de la croissance des effectifs renoue, depuis 2008, avec cette tendance de longue durée au resserrement de l'activité et des revenus.

Artistes et techniciens intermittents ne sont pas touchés de la même façon par ces évolutions. En 2009, un artiste intermittent déclare en moyenne 44 jours de travail annuel pour une rémunération brute de 9200 euros, et un technicien intermittent déclare 82 jours de travail annuel pour une rémunération moyenne de 16600 euros. Les artistes enregistrent donc des durées annuelles moyennes de travail et des volumes de rémunération très inférieurs à ceux des personnels techniques ; la part des indemnités chômage est aussi, de ce fait, beaucoup plus importante dans leur revenu global.

En vingt ans, la flexibilité de l'emploi s'est fortement accrue : les durées de travail se sont fractionnées en un nombre plus important de contrats de travail de courte durée. Là encore, les situations des artistes et des techniciens sont différentes : en 2009, un artiste déclare en moyenne 15 contrats de travail dans l'année contre 7 en 1990, pour une durée moyenne de 3 jours en 2009, contre 10 jours vingt ans plus tôt. Un technicien du spectacle, de son côté, déclare en moyenne 14 contrats de travail dans l'année contre 6 en 1990, pour une durée moyenne de 6 jours en 2009 , contre 14 jours vingt ans plus tôt.

\section{À paraître}

\section{CE 2014-3}

\section{La lente féminisation des professions culturelles}

Marie Gouyon, Frédérique Patureau

Octobre 2014

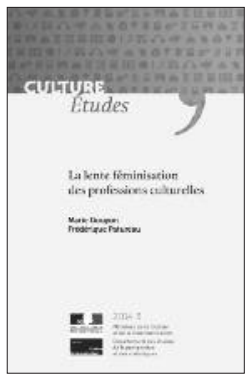

Depuis les années 1960, la croissance du taux d'activité féminine constitue l'une des transformations majeures du monde du travail. Cette progression ininterrompue et de grande ampleur conduit aujourd'hui, pour la première fois, à une répartition quasi paritaire des actifs : les femmes représentent $48 \%$ des actifs en emploi en 2011 contre $37 \%$ seulement en 1975. Les professions culturelles ne sont pas restées à l'écart de cette tendance de fond : la part des femmes y progresse de façon régulière et dans une proportion tout à fait comparable à celle observée dans l'ensemble de la population active.

Cette part demeure cependant, comme il y a vingt ans, inférieure à la moyenne nationale : $39 \%$ seulement de femmes dans les métiers culturels en 1991 et 43 \% en 2011.

Rapproché de l'implication privilégiée des femmes dans le champ artistique et culturel, ce constat peut sembler paradoxal. En effet, toutes les enquêtes relatives à la fréquentation des équipements culturels, aux pratiques artistiques et culturelles en amateur font le constat d'une surreprésentation féminine qui tend à s'amplifier notablement et continûment sur période longue, en France comme ailleurs.

Nature et durée des contrats, modalités d'organisation et quotité du temps de travail, l'étude fait le point sur la féminisation de l'emploi au sein des professions culturelles au cours des années 1990 et 2000. 


\section{Abstract}

\section{Twenty Years of Changing Employment Patterns in the Cultural Professions (1991-2011)}

Over the last twenty years, the number of people working in the cultural professions has undergone an unparalleled expansion, far outstripping that of the overall working population. In the early 1990 s as now, the profiles of individuals working in these professions and their jobs shared a number of common characteristics which set them apart from the rest of the working population, above and beyond the considerable diversity of jobs covered. In comparison with the working population as a whole, individuals in this group are on average younger, more likely to be male, considerably better qualified and more likely to be from the Paris area. What is notable about employment here is the considerable proportion of unsalaried workers, which has remained constant over the last twenty years (three times higher than that for the working population as a whole) and the much greater flexibility of the workforce (more short-term contracts, part-time working and the holding of multiple jobs).

The cultural professions have naturally been affected by the changes which have taken place within the world of work as a whole since the early 1990s. The continued feminisation of employment, for example, has occurred to just the same extent as it has across the working population as a whole, with the result that some traditionally very masculine cultural professions have seen their female workforce double in the space of twenty years.

Moreover, public policies encouraging decentralisation have led to a slight decrease in the number of Paris-based employees in these professions. Other trends observed across the working population as a whole, such as increasing levels of educational qualifications and an increasingly flexible workforce (two characteristics which have long been typical of the cultural employment field) have similarly continued to rise within this sector, reaching consistently higher levels than those seen within the working population as a whole.

Directeur de la publication : Xavier Niel

Responsable de la publication : Edwige Millery

\section{Retrouvez l'ensemble des publications du DEPS sur : http://www.culturecommunication.gouv.fr/Etudes-et-statistiques et sur http://www.cairn.info}

Le DEPS n'assurant pas de diffusion physique de ses collections de synthèse, nous vous proposons de vous informer régulièrement des parutions par message électronique.

Pour ce faire, merci de bien vouloir nous communiquer votre courriel à l'adresse contact.deps@culture.gouv.fr 
